\title{
Evaluación de las propiedades mecánicas de materiales compuestos elaborados a partir de cenizas volantes y polímeros reciclados
}

\author{
W. Aperador \\ J. Bautista** \\ A. E. Delgado***
}

Recibido: 06/05/2015 - Aceptado: 12/06/2015

\begin{abstract}
Resumen
En el presente artículo se evalúan las propiedades mecánicas de los materiales compuestos basados en cenizas volantes de carbón de la central termoeléctrica de Termozipa combinadas con los película extensible (Stretch film), polietilenos de baja densidad lineal de pos-consumo y polímero termoplástico parcialmente cristalino pos- industrial. Se obtuvieron mezclas variando el contenido de cenizas volantes de 0 a $50 \%$ en peso en cada uno de los tres materiales poliméricos, dentro de una máquina mezcladora tipo Brabender. Las propiedades mecánicas evaluadas fueron: resistencia a la tracción, dureza Shore D, y absorción de energía. Los resultados obtenidos indican que en todos los casos a medida que se agrega ceniza volante las propiedades mecánicas aumentan.

Palabras clave: polímeros reciclados, ceniza volante, material compuesto, propiedades mecánicas.
\end{abstract}

Correspondencia del autor: W. Aperador. Dr. Grupo de investigación Volta. Universidad Militar Nueva Granada, Bogotá, E-mail:g.ing.materiales@gmail.com

** Correspondencia del autor: J. Bautista. MSc. Grupo de investigación en tecnología cerámica. Universidad Francisco de Paula Santander, Cúcuta. E-mail: jbautistaruiz@yahoo.es

*** Correspondencia del autor: A. E. Delgado. MSc. Grupo de Ingeniería de Materiales. Universidad Militar Nueva Granada, E-mail: arnoldo.delgado@unimilitar.edu.co 


\section{Evaluation of mechanical properties of composite materials made from recycled fly ash and polymers}

\section{Abstract}

In this article evaluates the mechanical properties of composite materials based on fly ash and Coal-fired power plants Termozipa combined with the stretch film (stretch film), linear low density polyethylene post-consumer and partially thermoplastic polymer, crystalline Industrial post. Mixtures were obtained by varying the content of fly ash, 0 to $50 \%$ by weight in each of the three polymeric materials, within a machine type Brabender mixer. The mechanical properties evaluated were: tensile strength, Shore $\mathrm{D}$ hardness, and energy absorption, the results obtained indicate that in all cases, as fly ash is added, the mechanical properties increase.

Key words: Recycled polymers, fly ash, composite material, mechanical properties 


\section{INTRODUCCIÓN}

Los nuevos materiales de ingeniería buscan satisfacer todos los requerimientos mecánicos de diseño y costo, generando un menor impacto al ambiente durante su producción o en servicio [1-2]. Con los requerimientos de la industria de la construcción en el mundo, de emplear materiales cada vez más económicos que contribuyan con el sostenimiento del medio ambiente, se ha propiciado el desarrollo de materiales alternativos en la producción de paneles de construcción [3]. Dos tipos específicos de dichos materiales son los obtenidos a partir de polímeros reciclados y cenizas volantes [4].

La evolución de los materiales en la época actual se ha dado más rápido que en cualquier momento anterior en la historia. Nuevos polímeros, elastómeros, cerámicas y materiales compuestos están en desarrollo con formas de procesamiento que facilitan una oferta económica, con producción más reproducible frente a materiales convencionales [5-6]. También los materiales compuestos de matriz polimérica se han desarrollado en un nivel de producción comercial en la industria automotriz, de envasadoras, electrónica, aeroespacial, y de aparatos recreativos [7-10].

Por otra parte, el rápido crecimiento en el uso de los materiales constituidos por sistemas poliméricos multifásicos (mezclas y compuestos) está relacionado directamente con la disponibilidad de métodos que permitan controlar las interacciones físicas y químicas en su interface. Los compuestos que actúan como agentes interfaciales son denominados compatibilizadores para el caso de mezclas y agentes de acople en el compuesto. Las propiedades físicas y mecánicas eficaces están relacionadas con la presencia de una fase finamente dispersa con buena adhesión con la matriz y resistencia a la coalescencia [11-13].

Adicionalmente, las centrales termoeléctricas generan una alta tasa de ceniza volante, debido a que por cada tonelada de carbón pulverizado que se quema en una central termoeléctrica de carbón se producen aproximadamente unos $200 \mathrm{~kg}$ de cenizas volantes; el consumo del carbón pulverizado origina problemas ambientales debido a la acumulación de cenizas volantes en grandes depósitos en zonas próximas al lugar de consumo. En la mayoría de casos, se tiende a utilizar los residuos de combustión del carbón para reemplazar otros recursos naturales y por tanto, éste proceso en las térmicas ofrece algunos beneficios ambientales. Debido a la elevada producción a escala nacional y mundial de cenizas volantes es necesario el reciclaje de dicho producto; por ello actualmente, un mecanismo de control ambiental es su utilización en la fabricación de cemento [14-15].

Es de resaltar que el reciclaje de polímeros en Colombia se encuentra en una etapa inicial; sin embargo, en países como Alemania, Estados Unidos, Japón, Dinamarca, 
entre otros, se han desarrollado programas de tratamiento de residuos con el propósito de generar nuevos materiales. Entre estos se encuentra la Agencia de Protección Ambiental (EPA) de EE. UU. [16], que hizo un estudio de residuos sólidos urbanos (RSU) en el que muestra cómo los residuos poliméricos son el tercer componente más grande después del papel y residuos de material de construcción producidos en los RSU, que representan el $9 \%$ en peso. El porcentaje de reciclado de polímeros es solamente del $5 \%$ frente al $40 \%$ para el papel y el $30 \%$ de residuos de material de construcción, y puesto que el reciclado de polímeros requiere alta tecnología, los costos del proceso de reciclado se tornan elevados.

Es de destacar que el polietileno de baja densidad (Stretch film) utilizado en el hogar y en el embalaje pos-consumo; el polietileno de baja densidad lineal utilizado como empaque de bolsas de leche pos-consumo, y el polipropileno pos-industrial son tipos de polímeros que se encuentran en los RSU.

El objetivo de este estudio es el uso de polímeros reciclados y las cenizas volantes en la producción de un nuevo material compuesto, con el propósito de utilizarlo en el sector de la construcción civil. Puesto que los materiales utilizados en esta investigación son residuos sólidos, la utilización de ellos genera un beneficio en el medio ambiente.

\section{DESARROLLO EXPERIMENTAL}

\subsection{Materiales}

Los materiales poliméricos de pos-consumo y pos-industrial, fueron obtenidos de diferentes fuentes. El polietileno de baja densidad lineal fue reciclado de los empaques de bolsas de leche; el polietileno de baja densidad (Stretch film) es parte de la masa de residuos sólidos urbanos ya que combina usos varios en el hogar como protección y empaque, conservación de alimentos, trasteos o envíos, etc. El polipropileno proviene de los residuos de los procesos de fabricación de telas no tejidas. El carbón utilizado en la central termoeléctrica de Termozipa es triturado, pulverizado y posteriormente introducido dentro de la cámara de combustión. Los componentes orgánicos son oxidados y volatilizados durante la combustión mientras que una gran parte de la materia mineral es transformada en subproductos residuales sólidos: cenizas volantes y escorias. Las cenizas volantes están compuestas por partículas que debido a su tamaño pequeño son arrastradas por el flujo de gases que se generan durante la combustión del carbón. Para retener las cenizas volantes del flujo de gases que salen de la cámara de combustión y evitar que sean emitidas a la atmósfera, se emplea un sistema de retención denominado precipitador electrostático, el cual presenta una mayor eficiencia, por lo general superior al 99,5\% [17]. Las cenizas volantes se recogen en las tolvas 
de los precipitadores electrostáticos, desde donde son transportadas a lugares de almacenamiento, situados en balsas de decantación. Esta ceniza volante se caracteriza como clase F cenizas, según la norma ASTM especificación estándar C 618 [18]. Las partículas fueron principalmente las esferas, como se muestra en la fotomicrografía electrónica de barrido en la figura 1 .

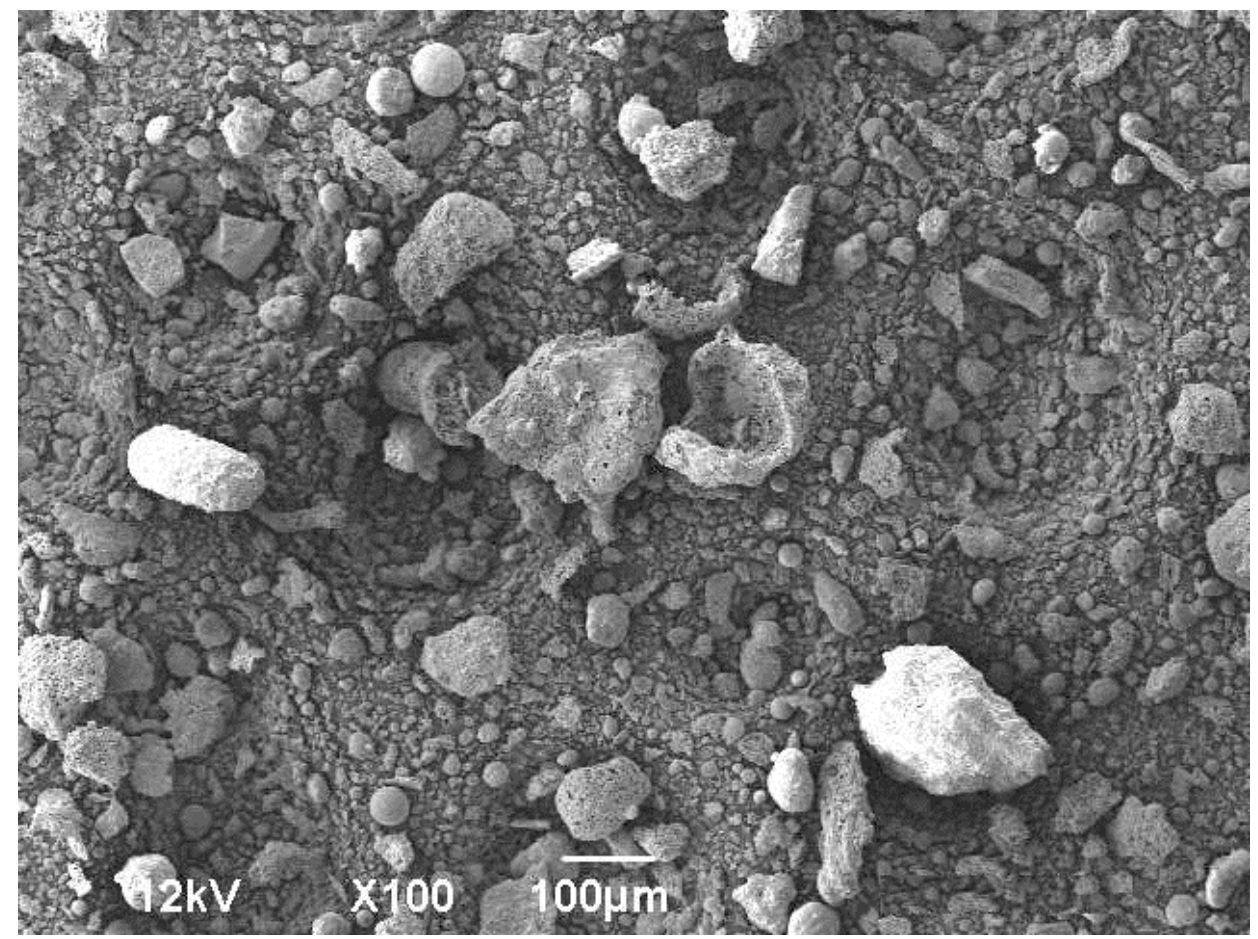

Figura 1. Micrografía de la ceniza volante,

tomada mediante un microscopio electrónico de barrido.

Fuente: los autores

\subsection{Detalles experimentales}

El procedimiento para la obtención de material compuesto se hace en tres etapas principalmente: (1) Pesaje de cada uno de los componentes (polímeros y ceniza volante), y a continuación la mezcla de polímeros y las cenizas volantes, (2) Calentamiento y mezcla de polímeros con las cenizas volantes para la elaboración de la muestra, y (3) Moldeo. El contenido de cenizas volantes varió de 0, 10, 20, 30, 40, y 50 \% en peso. El proceso de calentar y efectuar la mezcla fue hecho en una mezcladora tipo Brabender (figura 2, se observa la vista frontal donde se observan el sistema de mezclador y el contenedor en donde se fusionó el material compuesto), que es un equipo que cuenta 
con mecanismos de mezclado que genera movimientos de tipo difusión molecular, movimiento turbulento y convectivo, y mezclas de forma distributiva.

El mezclador se compone de contenedores, separadores, elementos de transmisión de potencia, sistemas de lubricación, contención de fluidos y de calentamiento. Además, cuenta con un sistema de control y lectura de los parámetros de temperatura y velocidad obtenidos mediante la tarjeta NI PCI 6221 de National Instruments; usando el software de instrumentación virtual Lab-VIEW ${ }^{\circ}$, la temperatura de procesamiento fue de $200{ }^{\circ} \mathrm{C}$, siendo $50 \mathrm{rpm}$ la velocidad en todos los casos.

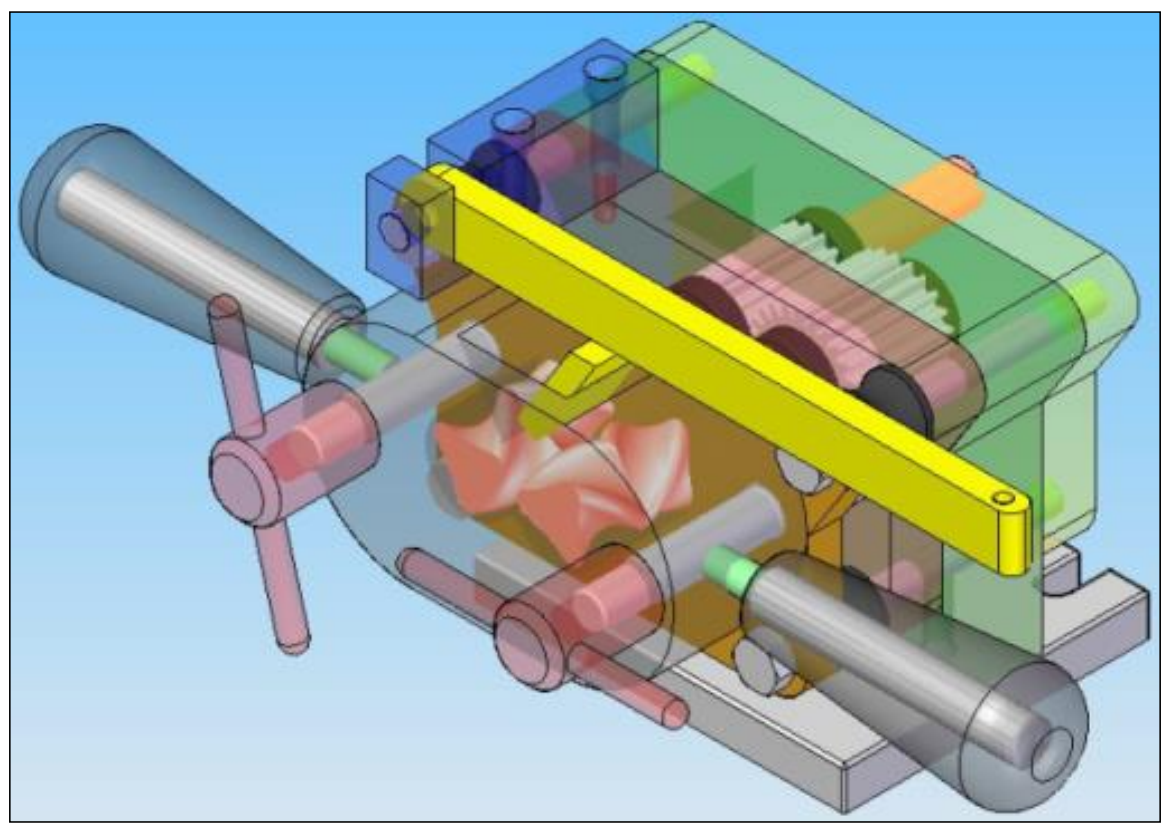

Figura 2. Mezcladora tipo Brabender

Las probetas para el análisis de tracción fueron elaboradas tomando como base la norma ASTM D 638 [19]; las probetas de impacto fueron fabricadas tomando como base la norma ASTM D 256 [20], y la dureza de los termoplásticos se hizo mediante la prueba Shore D) descrita en la norma ASTM D2240 [21].

\section{RESULTADOS}

\subsection{Prueba de tensión}

Los ensayos de tracción se hicieron a probetas con diferentes porcentajes de contenido de cenizas volantes $(0,10,20,30,40$, y $50 \%$ en peso) y polímeros tales como: polie- 
tileno de baja densidad (PEBD) denominado M1; polietileno de baja densidad lineal (PEBDL) reciclado de los empaque de bolsas de leche, denominados $\mathrm{M} 2 \mathrm{y}$ polipropileno pos-industrial de recorte de tela no tejidas, denominado M3.

Las propiedades mecánicas se analizaron a partir de ensayos tracción. Un aumento del contenido de cenizas volantes en las formulaciones aumenta notablemente la rigidez del material ya que se obtiene un aumento progresivo del valor del módulo de Young. Como se observa en las figuras 3 y 4, el aumento del módulo de Young fue de $32 \%$ y el $41 \%$.

La presencia del refuerzo de desecho industrial restringe la movilidad de las cadenas moleculares del polietileno, y contribuye con el incremento de rigidez a la de las formulaciones obtenidas [22]. El aumento de la rigidez de las formulaciones está en relación inversa con la capacidad de deformación del material, como se puede observar en las figuras 3 y 4 , ya que el alargamiento en el punto de rotura disminuye bruscamente al introducir cualquier porcentaje de ceniza volante. La resistencia a la tensión aumenta un $67 \%$ al agregarse un $50 \%$ de ceniza volante al polietileno de baja densidad en comparación con el material polimérico; este aumento considerable de resistencia a la tensión abre la posibilidad de utilizar este material en pisos industriales.

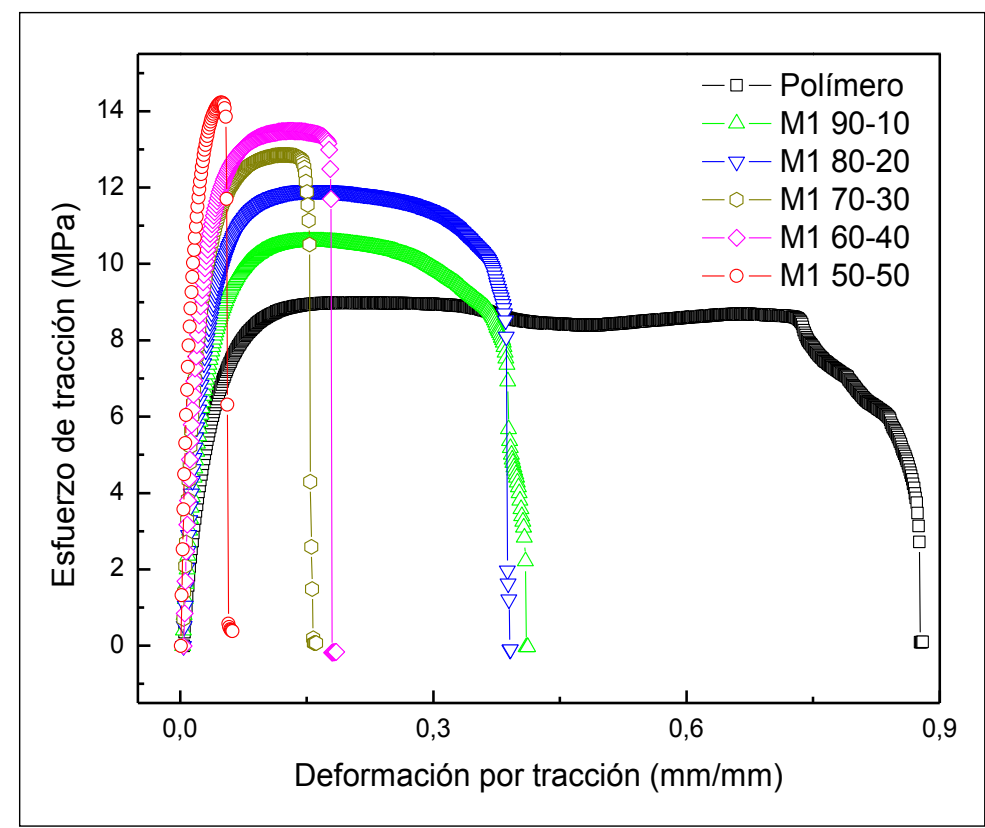

Figura 3. Polietileno de baja densidad (Stretch film) mezclado con ceniza volante en porcentajes del 0 al $50 \%$.

Fuente: los autores 
En la figura 4 se muestra el comportamiento del polietileno de baja densidad lineal (PEBDL), el cual, al igual que el polietileno de baja densidad (figura 3), aumenta la resistencia a la tensión en un $71 \%$ y disminuye el módulo elástico en un $54 \%$; el PEBDL presenta mejor resistencia a la tracción y mejor elongación que el PEBD. Al combinar el PEBDL con las cenizas volantes se observa un aumento en la resistencia a la tensión del $60 \%$ al mezclarla con el $50 \%$ de cenizas volantes.

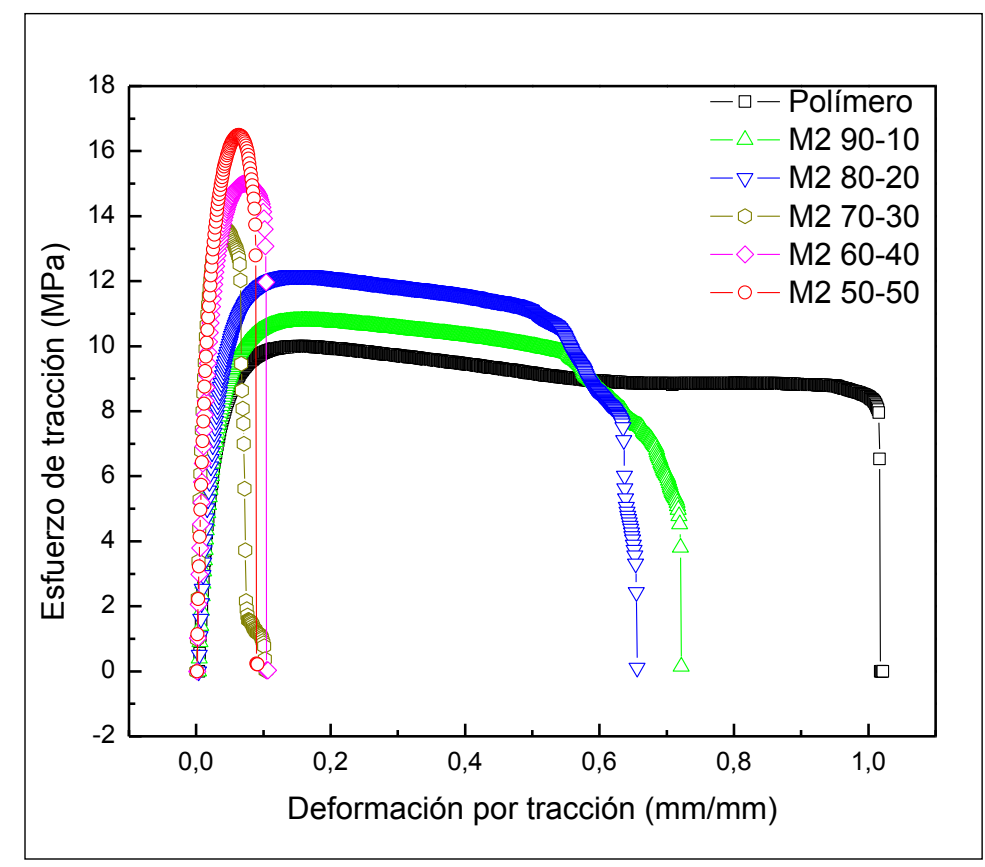

Figura 4. Polietileno de baja densidad lineal reciclado de los empaques de bolsas de leche, mezclado con ceniza volante en porcentajes del 0 al $50 \%$.

Fuente: los autores

En la figura 5 se observa el polipropileno pos-industrial, el cual genera un aumento elevado en la resistencia a la tensión en un $98 \%$, así como en el módulo elástico un 89 $\%$, lo que indica el mejor comportamiento de los sistemas evaluados. 


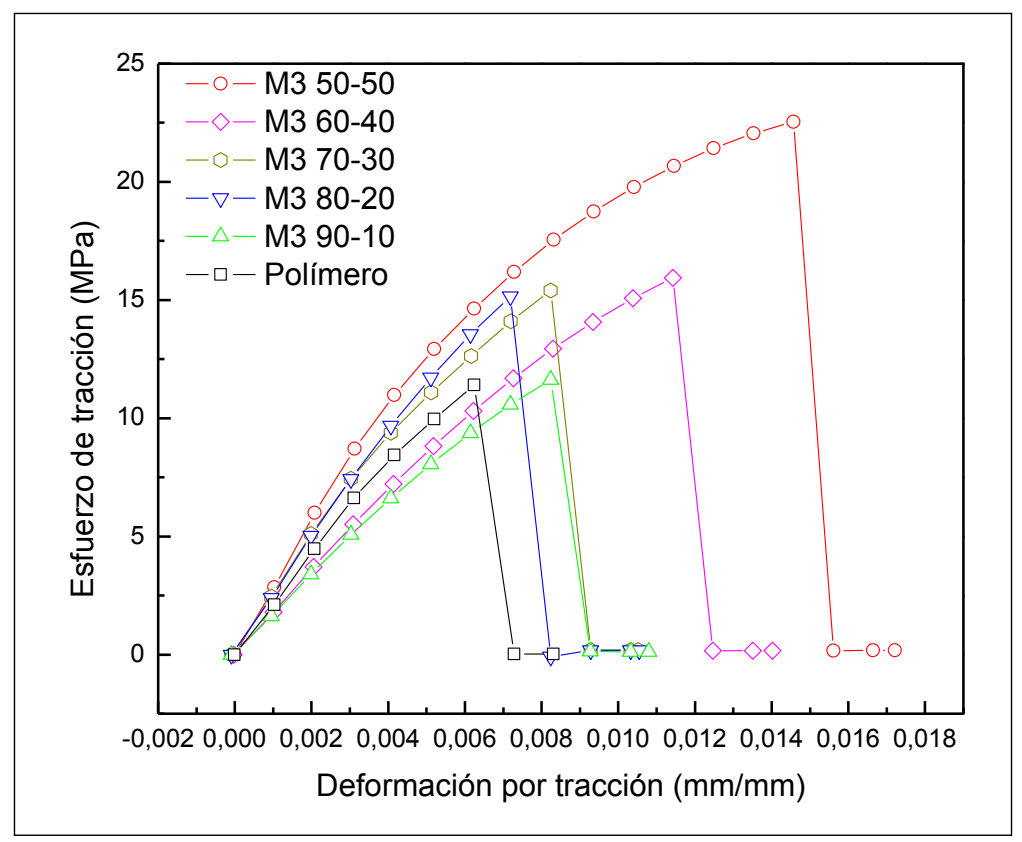

Figura 5. Polipropileno post industrial de recorte de tela no tejidas mezclado con ceniza volante en porcentajes del 0 al $50 \%$.

Fuente: los autores

\subsection{Dureza Shore D}

Cuando se estudian comparativamente los valores de dureza Shore D para las diferentes mezclas (figura 6), se observa que aumenta al disminuir el porcentaje de las tres diferentes clases de polímeros evaluados.

La dureza de la mezcla polipropileno pos-industrial de recorte de tela no tejida mezclado con ceniza volante es la de mayor valor, lo cual parece evidenciar una creciente interacción entre los componentes de la mezcla, y conlleva un aumento en la dureza superficial. Los polietilenos de baja densidad y lineal muestran un aumento similar en los primeros porcentajes, y posteriormente el M1 se mantiene en incremento de la resistencia, mientras el M2 genera un aumento similar al polipropileno [23]. 


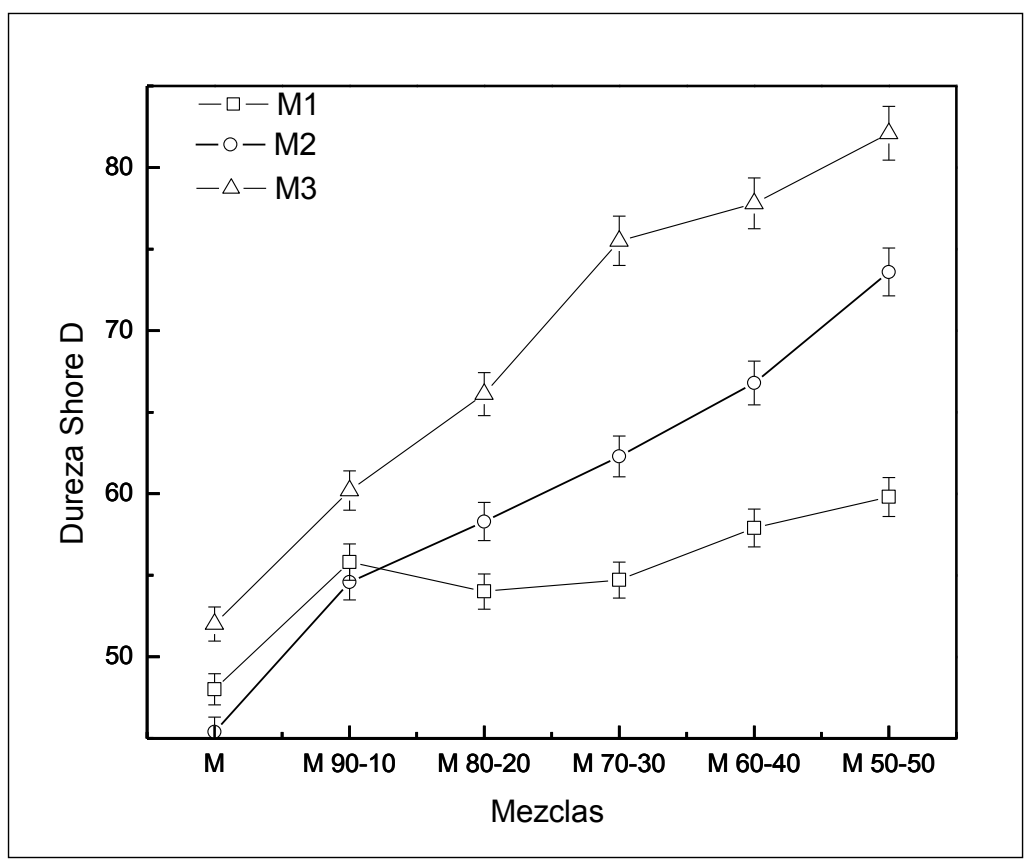

Figura 6. Variación de la dureza Shore D del Polietileno de baja densidad (M1), Polietileno de baja densidad lineal (M2) y Polipropileno post industrial de recorte de tela no tejidas (M3) mezclados con ceniza volante en porcentajes del 0 al $50 \%$.

\subsection{Energía absorbida}

El polipropileno pos-industrial tiene buenas propiedades al impacto, ya que es capaz de absorber una gran cantidad de energía. La resistencia al impacto de las formulaciones con ceniza volante sufre un aumento al aumentar la cantidad de dicha carga, tal como se aprecia en la figura 7. Este aumento puede estar causado por una buena adhesión entre el polipropileno y la ceniza volante. Las mezclas de polietileno de baja densidad PEBD y PEBDL generan valores similares con bajos contenidos de ceniza volante. $\mathrm{La}$ mezcla de PEBDL genera mayores valores de energía absorbida al aumentar la carga de ceniza volante. 


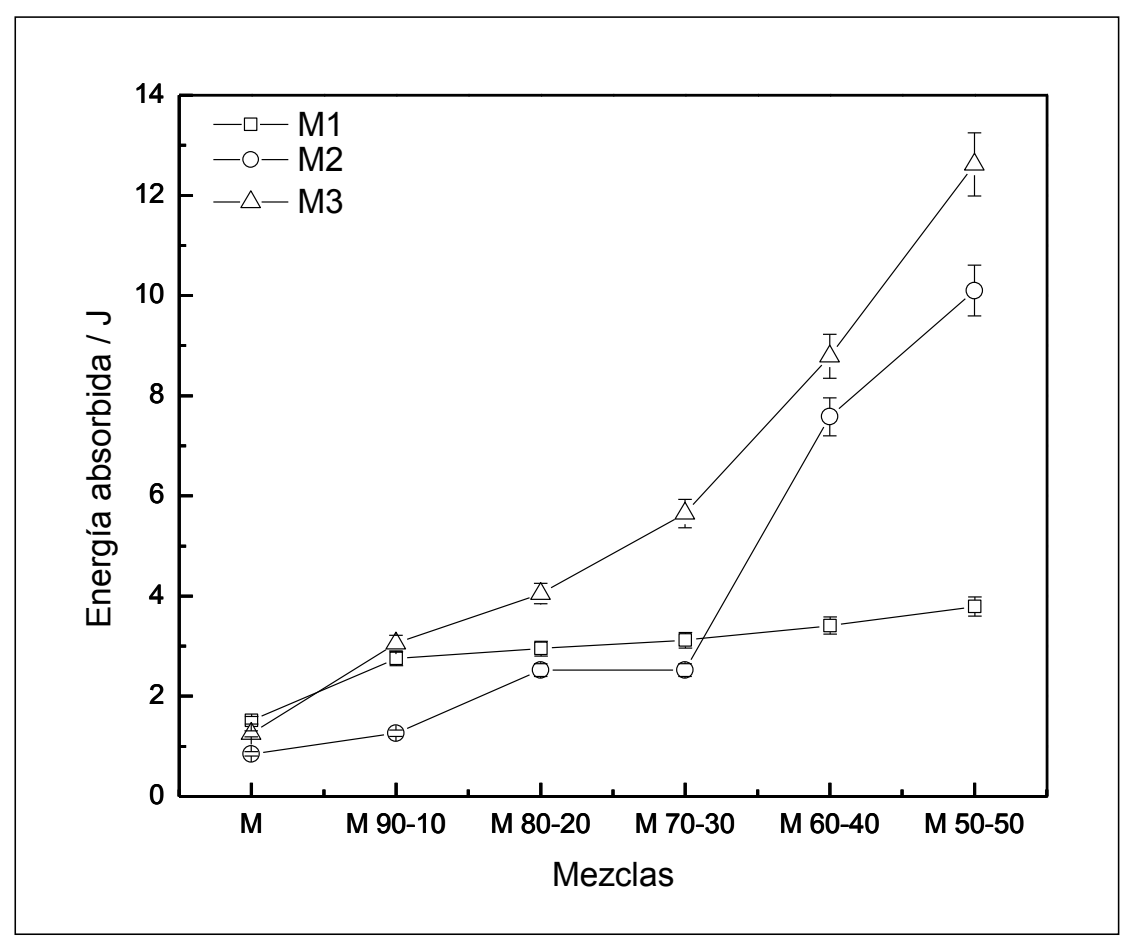

Figura 7. Variación de la energía absorbida del Polietileno de baja densidad (M1), Polietileno de baja densidad lineal (M2) y polipropileno post industrial de recorte de tela no tejidas (M3) mezclados con ceniza volante en porcentajes del 0 al $50 \%$.

Fuente: los autores

\section{CONCLUSIONES}

La ceniza volante es un buen refuerzo para polímeros reciclados. Se obtiene un material con un aspecto similar al polímero comercial con mejores propiedades que el material sin refuerzo, llegando incluso a duplicar el valor del módulo de Young del polietileno de baja densidad y del polipropileno pos-industrial, al añadir un $50 \%$ de ceniza volante. Las resistencias a la tracción, la dureza, la densidad y a la temperatura aumentan con respecto a los polímeros sin carga.

Otro aspecto destacable de estas formulaciones es que se pueden transformar mediante inyección de manera análoga al polietileno sin refuerzos.

\section{AGRADECIMIENTOS}

Producto derivado del proyecto ING. 1572, financiado por la Vicerrectoría de Investigaciones de la UMNG, vigencia 2015. 


\section{REFERENCIAS}

[1] A.K. Sahani, P.K. Jain, Satish C. Sharma, J.K. Bajpai, "Design Verification through Tolerance Stack up Analysis of Mechanical Assembly and Least Cost Tolerance Allocation", Procedia Mater. Sci, Vol. 6, n. ${ }^{\circ}$ 1, pp. 284-295, 2014.

[2] R. Danzer, "On the relationship between ceramic strength and the requirements for mechanical design”, J. Eur. Ceram. Soc, Vol. 34, n. ${ }^{\circ}$ 15, pp. 3435-3460. 2014.

[3] G. Andreadis, G. Fourtounis, K-D. Bouzakis, "Collaborative design in the era of cloud computing”, Adv. Eng. Software, Vol. 81, n. ${ }^{\circ}$ 3, pp. 66-72, 2015

[4] J. Guru Jawahar, C. Sashidhar, I.V. Ramana Reddy, J. Annie Peter, "Design of cost-effective M 25 grade of self compacting concrete” Mater. Des, Vol. 49, pp. 687-692, 2013.

[5] Y. L. Xu and K. L. Reifsinder," Micromechanical modelling of composite compressive strength", J. Compos Mate, vol. 27, n. ${ }^{\circ}$ 6, pp. 572-588, 1993.

[6] J. Kenny, "Joining Technologies for Polymeric Matrix Composites: Present and Future. COMPOSIT workshop" on Joining Technologies for Composite Transportation Structures, Orbassano, 2003.

[7] A. S. Argon, "Fracture of composites", Treatise of material science and technology vol. 1, New York Academic Press, pp. 89-95, 1972.

[8] D. C. Lagoudas and A.M. Saleh, "Compressive failure due to kinking of fibrous composites", J. Compos Mater., vol. 27, n. ${ }^{\circ}$ 1, pp. 83-106, 1993.

[9] J. Recasens, "Nuevas Aplicaciones de los Materiales Compuestos en la Construcción", $16^{a}$ Jornadas de Materiales Compuestos/Plásticos Reforzados, Barcelona, pp. 25-29, 2002.

[10] J. Niklewicz, G. D. Sims, “Size effects in composite materials", Structural Integrity and Performance Project CPD1, National Physical Laboratory, NPL Report MATC (A) 74, January 2002.

[11] J. Milgrom, "Polyethylene terephthalate (PET)". Plastics Recycling: Products and Processes Ehrig RJ, editor, New York: Hanser, pp. 45-71, 2005.

[12] K.S. Rebeiz, D. W. Fowler and D. R. Paul, "Strength properties of unreinforced and reinforced polymer concrete using recycled PET”, Polym. Recycl, vol. 2, pp. 133-139, 1996.

[13] C. Alkan, M. Arslan, M. Cici, M. Kaya and M. Aksoy, "A study on the production of a new material from fly ash and polyethylene", Resour. Conserv. Rec., vol. 13, pp. 147-154, 1995.

[14] M. Criado, A. Palomo and A. Fernández-Jiménez, "Alkali activation of fly ashes", Part 1. Effect of curing conditions on the carbonation of the reaction products, Fuel, vol. 84, pp. 2048-2054, 2005.

[15] D.M. Bastidas, A. Fernández-Jiménez, A. Palomo and J.A. González, "A study on the passive state stability of steel embedded in activated fly ash mortars", Corros. Sci. vol. 50, pp. 1058$1065,2008$. 
[16] US EPA, "Characterization of municipal solid waste in the United States: 2006 Update”, EPA 540-R-67-034. Pp. 125-129, 2007.

[17] I. SMITH, “Trace Elements from Coal Combustion: Emissions", IEACR/01. IEA Coal Research, pp. 871987.

[18] ASTM C618 - 08a, "Standard Specification for Coal Fly Ash and Raw or Calcined Natural Pozzolan for Use in Concrete", West Conshohocken, PA, American Society for Testing and Materials, 2008.

[19] ASTM D 256-05, "Standard Test Methods for Determining the Izod Pendulum Impact Resistance of Plastics", West Conshohocken, PA, American Society for Testing and Materials, 2005.

[20] ASTM D 638- 03, “Standard Test Method for Tensile Properties of Plastics”, West Conshohocken, PA, American Society for Testing and Materials, 2003.

[21] ASTM D2240 - 05, “Standard Test Method for Rubber Property— Durometer Hardness", West Conshohocken, PA, American Society for Testing and Materials, 2010.

[22] H. F. R. Ferreto, A. C. F. Oliveira, L. F. C. P. Lima, D. F. Parra, A. B. Lugão "Thermal, tensile and rheological properties of linear low density polyethylene (LLDPE) irradiated by gammaray in different atmospheres" Radiat. Phys. Chem., Vol. 81, n. ${ }^{\circ}$ 8, pp. 958-961, 2012

[23] T. Boronat, V. Fombuena, D. García-Sanoguera, L. Sánchez-Nacher, R. Balart “Development of a biocomposite based on green polyethylene biopolymer and eggshell" Mater. Des, Vol. 68, Pages 177-185, 2015. 